\title{
Prevalence of Social Determinants of Health and Associations of Social Needs Among United States Adults, 2011-2014
}

\author{
Eun Ji Kim, MD, $M S^{\top} \odot$, Sara Abrahams, $B A^{\top}$, Omolara Uwemedimo, $M D, M P H^{2}$, and \\ Joseph Conigliaro, MD, MPH
}

'Division of General Internal Medicine, Donald and Barbara Zucker School of Medicine at Hofstra/Northwell , Lake Success , NY , USA; ${ }^{2}$ Department of Pediatrics, Donald and Barbara Zucker School of Medicine at Hofstra/Northwell , Lake Success, NY , USA.

J Gen Intern Med 35(5): 1608-9

DOI: $10.1007 / \mathrm{s} 11606-019-05362-3$

(c) Society of General Internal Medicine 2019

\section{INTRODUCTION}

Social determinants of health (SDHs) are conditions arising from where people are born, live, learn, work, and age and account for $80 \%$ of health outcomes. ${ }^{1,2}$ The prevalence of different SDH has been examined, but most studies used community data or examined one specific SDH need. ${ }^{3,4}$ To better understand the significance of the SDH at a national level, it is important to examine the prevalence of different SDHs and how individual SDH needs correlate with one another. Using the National Health and Nutrition Examination Survey (NHANES), we examined the prevalence of low education, low income, no health insurance, food insecurity, poor housing, and limited/no employment individually, and whether the presence of one SDH need is associated with the presence of another.

\section{METHODS}

We used the 2011-2014 NHANES, a biennial nationally representative survey. ${ }^{5}$ We included all adults 18 years or older $(n=11,977)$ and excluded 160 participants with invalid/ incomplete responses to covariates. Six domains of SDH were identified: low education, low income, no health insurance, food insecurity, poor housing, and no/limited employment. We defined low education as having lower than a high school degree and low income as either having family income equal to or less than $100 \%$ of the Federal Poverty Level or individual income equal to or less than $\$ 34,999$. Those who answered "often true" or "sometimes true" to questions including "worried about food not lasting" or "food not lasting" were identified as having food insecurity. Participants were categorized as "poor housing" if the ratio of the number of household members to number of rooms was greater than 2 or if the living

Received July 24, 2019

Accepted September 12, 2019

Published online November 20, 2019 arrangement was neither owned/being bought nor rented. Lastly, we grouped those who were looking for work or did not work in the past week (excluding those who attended school or were retired) as no/limited employment. For our statistical analyses, we incorporated multi-stage stratification sampling design and weights. First, we performed descriptive analyses of all participants. We examined the prevalence of each SDH need (low education, low income, no health insurance, food insecurity, poor housing, and no/limited employment) and the prevalence of multiple SDH needs. Next, we calculated the Rao-Scott chi-square test to examine associations between different SDH needs. All statistical analyses were conducted using SAS 9.4 (SAS Institute Inc., Cary, NC, USA). The Feinstein Institute Institutional Review Board granted exempt status.

\section{RESULTS}

There were 11,817 survey participants (weighted sample of 463 million US adults) and more than half ( $54.4 \%$, weighted) of the population had one or more SDH need (Table 1). The prevalence of different SDH needs included the following: $16.6 \%$ with low education, $21.8 \%$ with low income, $19.0 \%$ without health insurance, $23.0 \%$ with food insecurity, $3.3 \%$ with poor housing, and $21.0 \%$ with no or limited employment. Percentages of patients decreased as the number of SDH needs increased: $24.9 \%$ had one SDH need while $1.0 \%$ had five or more SDH needs. Next, we examined associations among race/ ethnicity and various SDH needs. All SDH needs were significantly associated with each other ( $p$ values $<0.05$ ) except that poor housing was not associated with either race/ethnicity ( $p$ value $=0.46$ ) or low education $(p$ value $=0.33$ )

\section{DISCUSSION}

Social determinants of health are prevalent among American adults, with prevalence varying by domain. The presence of one SDH need is frequently associated with the presence of others, suggesting systematic strategies are needed to address and untangle the interrelationship among different SDH needs. 
Table 1 Survey Participants' Characteristics (Weighted \%)

\begin{tabular}{ll}
\hline \hline Characteristics & $\begin{array}{l}\text { Weighted } \\
\text { \% }\end{array}$ \\
\hline Race/ethnicity & \\
White & 65.8 \\
Black & 11.5 \\
Hispanic & 14.7 \\
Asian & 5.2 \\
Other & 2.7 \\
Male & 48.2 \\
Age group & \\
18-34 & 30.1 \\
35-49 & 26.3 \\
$50-64$ & 26.1 \\
65+ & 17.6 \\
Social determinants of health & \\
Low education (less than high school) & 16.6 \\
Low income (less than 100\% FPL or <35K for & 21.8 \\
individual) & \\
No health insurance & 19.0 \\
Food insecurity & 23.0 \\
Poor housing & 3.3 \\
No/limited employment & 21.0 \\
Number of social needs present & \\
0 & 45.6 \\
1 & 24.9 \\
2 & 15.1 \\
3 & 9.1 \\
4 & 4.4 \\
$5+$ & 1.0 \\
\hline
\end{tabular}

Limitations of the study included restricted number of SDHs arising from secondary data and use of self-reported information. This demonstrates the importance of identifying interventions that could impact multiple domains of SDH needs and suggests a need for further investigation into whether or not interventions within one domain demonstrate an effect on others. $^{6}$
Corresponding Author: Eun Ji Kim, MD, MS; Division of General Internal MedicineDonald and Barbara Zucker School of Medicine at Hofstra/Northwell , 2001 Marcus Avenue Suite S160 , Lake Success , NY11042,USA (e-mail: ekim7@northwell.edu).

\section{Compliance with Ethical Standards:}

Conflict of Interest: The authors declare that they do not have a conflict of interest.

\section{REFERENCES}

1. Marmot M. Social determinants of health inequalities. Lancet. 2005;365(9464): 1099-1104.

2. Schroeder SA. Shattuck Lecture. We can do better-improving the health of the American people. N Engl J Med 2007;357(12):1221-1228.

3. Uwemedimo OT, May H. Disparities in Utilization of Social Determinants of Health Referrals Among Children in Immigrant Families. Front Pediatr 2018;6:207.

4. Kohn MJ, Bell JF, Grow HM, Chan G. Food insecurity, food assistance and weight status in US youth: new evidence from NHANES 2007-08. Pediatr Obes 2014;9(2):155-166.

5. National Health and Nutrition Examination Survey (NHeANES). 20112015; http://www.cdc.gov/nchs/nhanes/. Accessed July 11, 2019.

6. Gottlieb L, Cottrell EK, Park B, Clark KD, Gold R, Fichtenberg C. Advancing Social Prescribing with Implementation Science. J Am Board Fam Med 2018;31(3):315-321.

Publisher's Note Springer Nature remains neutral with regard to jurisdictional claims in published maps and institutional affiliations. 